Honam Mathematical J. 36 (2014), No. 2, pp. 425-434

http://dx.doi.org/10.5831/HMJ.2014.36.2.425

\title{
A GENERALIZATION OF A SEQUENTIAL SPACE AND RELATED SPACES
}

\author{
Woo Chorl Hong ${ }^{\dagger}, *$ And Seonhee Kwon
}

\begin{abstract}
In this paper, we introduce a new concept of a countably sequential space which is a generalization of a sequential space and study some properties of a countably sequential space and relations among the space and related spaces.
\end{abstract}

\section{Introduction}

All spaces considered here are always assumed to be infinite $T_{1}$. Our terminology is standard and follows [5] and [15]. For each subset $A$ of a topological space $X$, we use $\bar{A}$ for the closure of $A$ in $X$. Let $\mathbb{N}$ denote the set of positive integers and $\left(x_{n}: n \in \mathbb{N}\right.$ )(briefly $\left(x_{n}\right)$ ) a sequence of points of a set.

A space $X$ has unique sequential limits [6] iff for every sequence of points of $X$ may converge to at most one limit. It is well-known that every topological space with unique sequential limits lies between $T_{1}$ and Hausdorff.

Let us recall a sequential space and some related spaces which are generalizations of a first countable space.

A space $X$ is sequential $[6,8]$ iff for each subset $A$ of $X$ which is not closed in $X$, there exist $x \in \bar{A} \backslash A$ and a sequence $\left(x_{n}\right)$ of points of $A$ such that $\left(x_{n}\right)$ converges to $x$ in $X$. A space $X$ is Fréchet-Urysohn $[1,8]$ (also called Fréchet [6,7] or closure sequential [15]) iff for each subset $A$ of $X$ and each $x \in \bar{A}$, there exists a sequence $\left(x_{n}\right)$ of points of $A$ such that $\left(x_{n}\right)$ converges to $x$ in $X$. A space $X$ is countably Fréchet-Urysohn [8] iff

Received April 1, 2014. Accepted May 21, 2014.

2010 Mathematics Subject Classification. 54A20, 54D20, 54D55, and 54E25.

Key words and phrases. sequential, countably sequential, countable tightness, Fréchet-Urysohn, AP, countably AP, WAP, WACP, and sequentially compact.

${ }^{\dagger}$ This work was supported by a 2-Year Research Grant of Pusan National University.

${ }^{*}$ Corresponding author 
for each countable subset $A$ of $X$ and each $x \in \bar{A}$, there exists a sequence $\left(x_{n}\right)$ of points of $A$ such that $\left(x_{n}\right)$ converges to $x$ in $X$. A space $X$ is $A P$ (standing for Approximation by Points) [3] (also called Whyburn [11]) iff for each non-closed subset $A$ of $X$ and each $x \in \bar{A} \backslash A$, there exists a subset $B$ of $A$ such that $\bar{B}=B \cup\{x\}$. A space $X$ is $W A P$ (standing for Weak Approximation by Points) [2] (also called weakly Whyburn [11]) iff for each subset $A$ of $X$ which is not closed in $X$, there exist $x \in \bar{A} \backslash A$ and a subset $B$ of $A$ such that $\bar{B}=B \cup\{x\}$. A space $X$ is countably $A P$ [8] iff for each countable non-closed subset $A$ of $X$ and each $x \in \bar{A} \backslash A$, there exists a subset $B$ of $A$ such that $\bar{B}=B \cup\{x\}$. A space $X$ is $A C P$ (standing for Approximation by Countable Points) [8] iff for each non-closed subset $A$ of $X$ and each $x \in \bar{A} \backslash A$, there exists a countable subset $B$ of $A$ such that $\bar{B}=B \cup\{x\}$. A space $X$ is $W A C P$ (standing for Weak Approximation by Countable Points) [8] iff for each subset $A$ of $X$ which is not closed in $X$, there exist $x \in \bar{A} \backslash A$ and a countable subset $B$ of $A$ such that $\bar{B}=B \cup\{x\}$. A space $X$ has countable tightness $[1,10]$ (also called closure countable [15]) iff for each subset $A$ of $X$ and each $x \in \bar{A}$, there exists a countable subset $B$ of $A$ such that $x \in \bar{B}$.

It is well-known that the following diagram exhibits the general relationships among the properties mentioned above except for three implications 1, 2 and 3 and if $X$ has unique sequential limits, then the three implications hold.

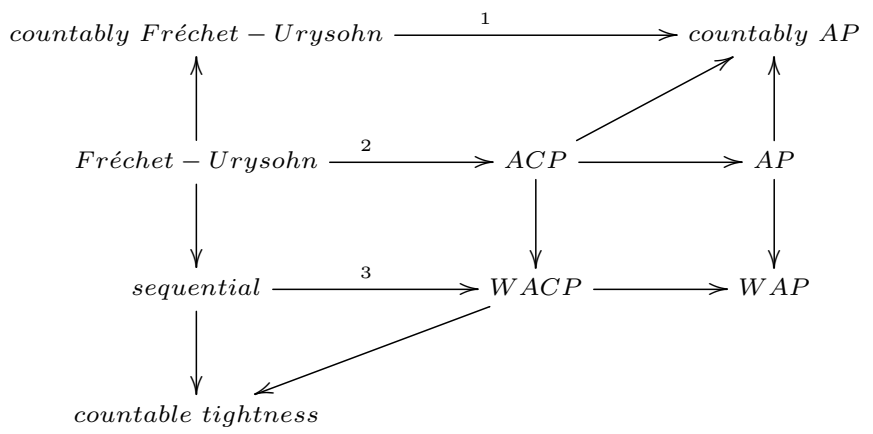

Several authors (see $[1,2,3,4,6,8,10,11,13]$ ) studied some properties of a sequential space and relations between a sequential space and related spaces. In particular, in $[6,7,8,9,13]$, the authors showed some sufficient conditions for a sequential space to be Fréchet-Urysohn.

In this paper, we introduce a new concept of a countably sequential space which is a generalization of a sequential space and study some properties of a countably sequential space and relations among the space 
and related spaces. First, we show that every countably sequential and WACP space is sequential and every countably sequential and countably AP space having countable tightness is Fréchet-Urysohn. And as corollaries, we obtain that every sequential and AP space is Fréchet-Urysohn ([1, Corollary 2.2(6)] and [13, Proposition 2.1(6)]) and in the class of countably sequential spaces having countable tightness and unique sequential limits, the five properties: Fréchet-Urysohn, countably FréchetUrysohn, ACP, AP, and countably AP coincide. We prove that a topological space $X$ is countably compact and countably sequential iff $X$ is sequentially compact, and obtain that every countably compact and sequential space is sequentially compact ([1, P.53, Prop 3], [6, Proposition 1.10] and $[14$, p.590, 5.3]) as a corollary. We study some necessary and sufficient conditions for a countably sequential space to have unique sequential limits. Finally, we introduce a new property $(*)$ of a topological space which is another generalization of a sequential space and show that the property $(*)$ is a necessary and sufficient condition for a topological space $X$ having countable tightness to be sequential.

\section{Results}

We begin by introducing countably sequential spaces.

Definition 2.1. A topological space $X$ is countably sequential iff for each countable subset $A$ of $X$ which is not closed in $X$, there exist $x \in \bar{A} \backslash A$ and a sequence $\left(x_{n}\right)$ of points of $A$ such that $\left(x_{n}\right)$ converges to $x$ in $X$.

It is clear that the following statements are true:

(1) Every sequential space is countably sequential.

(2) Every countable and countably sequential space is sequential.

(3) If every countable subset of a topological space $X$ is closed, then $X$ is countably sequential.

(4) Every sequentially compact space is countably sequential.

(5) Being countably sequential, being WAP and having countable tightness are independent (even though Hausdorff).

Obviously, the converses of (2) and (3) are not true. By the following Example 2.2 and Remark 2.3(1), we know that (5) is true and the converses of (1) and (4) are not true.

Example 2.2. (1) The space of ordinals $X=\left[0, \omega_{1}\right]$, where $\omega_{1}$ is the first uncountable ordinal, is a Hausdorff compact and WAP space. But, it does not have countable tightness and hence not sequential (see [8] 
and [12]). It is well-known that $\left[0, \omega_{1}\right)$ is a first countable space (and hence sequential) and for each countable subset $A$ of $X, \omega_{1} \notin \bar{A} \backslash A$. Hence, we have easily that $X$ is countably sequential.

(2) Let $X=\{(0,0)\} \cup(\mathbb{N} \times \mathbb{N})$. We define a topology $\tau$ on $X$ by for each $(m, n) \in X-\{(0,0)\},\{(m, n)\} \in \tau$ and $(0,0) \in U \in \tau$ iff for all but a finite number of integers $m$, the sets $\{n \in \mathbb{N}:(m, n) \notin U\}$ are each finite. Thus each point $(m, n) \in X-\{(0,0)\}$ is isolated and each open neighborhood of $(0,0)$ contains all but a finite number of points in each of all but a finite number of columns. Then the space $X$ is Hausdorff WAP having countable tightness (see [8]). Note that $\{(0,0)\}=\overline{\mathbb{N}} \times \mathbb{N} \backslash(\mathbb{N} \times \mathbb{N})$, but there does not exist a convergent sequence of points of $\mathbb{N} \times \mathbb{N}$ (see $[12$, p.54, 26(3)]). Thus, $X$ is not countably sequential and hence not sequential.

(3) Let $X=\mathbb{R}$, where $\mathbb{R}$ denotes the set of all real numbers and $\tau_{1}$ the usual topology on $\mathbb{R}$. Let $\tau=\left\{O \backslash K: O \in \tau_{1}, \mathrm{~K}\right.$ is a countable subset of $X\}$. Then the space $X$ endowed with the topology $\tau$ is Hausdorff and every countable subset of $X$ is closed (see [12] and [8]). It follows directly that $X$ is countably sequential and it does not have countable tightness. Moreover, in [4, Example 2.5], M. H. Cho, J. Kim and M. A. Moon showed that $X$ is not WAP.

(4) The real line $\mathbb{R}$ with the usual topology is sequential and hence countably sequential, but not sequentially compact.

Remark 2.3. (1) In [3, Theorem 2.1], A Bella and I. V. Yaschenko showed that there is a countable non WAP space. Hence, we have that a space having countable tightness need not be WAP in general.

(2) It is clear that the Stone- $\breve{C}$ ech compactification $\beta \mathbb{N}$ of the space $\mathbb{N}$ with the discrete topology is not countably sequential. Thus we have that every compact space need not be countably sequential.

We now show some properties of a countably sequential space and relations among the space and related spaces.

Theorem 2.4. Every closed subspace of a countably sequential space is countably sequential.

Proof. Let $A$ be a closed subspace of a countably sequential space $X$ and $C$ a countable subset of $A$ with $\bar{C}^{A} \backslash C \neq \emptyset$, where $\bar{C}^{A}$ is the closure of $C$ in the subspace $A$. Then since $A$ is closed in $X, \bar{C}=\bar{C}^{A}$. Hence, since $X$ is countably sequential, there exist $x \in \bar{C} \backslash C$ and a sequence $\left(x_{n}\right)$ of points of $C$ such that $\left(x_{n}\right)$ converges to $x$ in $X$. By closedness of $A$ in $X,\left(x_{n}\right)$ converges to $x$ in $A$. Thus, $A$ is countably sequential. 
Remark 2.5. (1) Note that in Example 2.2(1), the subspace $\left[0, \omega_{1}\right)$ of $X$ is countably sequential, but not closed. Hence, we know that a countably sequential subspace of a countably sequential space need not be closed in general.

(2) By the following Example, we have that countable sequentiality is not hereditary.

Example 2.6. Let $X$ be the set consisting of pairwise distinct objects of the following three types: Points $x_{m n}$ where $m, n \in \mathbb{N}$, points $y_{n}$ where $n \in \mathbb{N}$, and a point $z$. We set $V_{k}\left(y_{n}\right)=\left\{y_{n}\right\} \cup\left\{x_{m n}: k \leq m\right\}$ and let $\gamma$ denote the set of subsets $W$ of $X$ such that $z \in W$ and there exists a positive integer $p$ such that $V_{1}\left(y_{n}\right) \backslash W$ is finite and $y_{n} \in W$ for all $n \geq p$. The collection

$$
\beta=\left\{\left\{x_{m n}\right\}: m, n \in \mathbb{N}\right\} \cup \gamma \cup\left\{V_{k}\left(y_{n}\right): k, n \in \mathbb{N}\right\}
$$

is a base of a topology on $X$. Then the space $X$ endowed with the topology generated by the base $\beta$ is sequential, but not Fréchet-Urysohn (see [1, p.13, Example 13]). Hence, $X$ is countably sequential. Let $Y=X \backslash\left\{y_{n}: n \in \mathbb{N}\right\}$. Then clearly $\bar{Y}=X$ and hence $Y$ is not closed in $X$. Obviously, $\left\{x_{m n}: m, n \in \mathbb{N}\right\}$ is a countable subset of $Y$ and $\left\{x_{m n}: m, n \in \mathbb{N}\right\} \backslash\left\{x_{m n}: m, n \in \mathbb{N}\right\}=\{z\}$, but there does not exist a sequence $\left(s_{n}\right)$ of points of $\left\{x_{m n}: m, n \in \mathbb{N}\right\}$ such that $\left(s_{n}\right)$ converges to $z$ in the subspace $Y$. Thus, $Y$ is not countably sequential.

Next we study some sufficient conditions for a countably sequential space to be sequential or Fréchet-Urysohn and related topics.

Theorem 2.7. Every countably sequential and WACP space is sequential.

Proof. Let $A$ be a subset of a countably sequential and WACP space $X$ with $\bar{A} \backslash A \neq \emptyset$. Then since $X$ is WACP, there exist $x \in \bar{A} \backslash A$ and a countable subset $C$ of $A$ such that $\bar{C}=C \cup\{x\}$. Since $X$ is countably sequential, there exists a sequence $\left(x_{n}\right)$ of points of $C$ such that $\left(x_{n}\right)$ converges to $x$. Thus, $X$ is sequential.

From Theorem 2.7 above, we have the following corollaries.

Corollary 2.8. Every countably sequential and WAP space having countable tightness is sequential.

Proof. Note that a topological space $X$ is WACP iff $X$ is a WAP space having countable tightness. Hence, by Theorem 2.7, it holds. 
By Example 2.2(2), we know that every WAP space having countable tightness need not be sequential.

From Theorem 2.7 and Corollary 2.8, we have immediately the following corollary and hence we omit the proof.

Corollary 2.9. (1) For a topological space $X, X$ is sequential iff $X$ is countably sequential and WAP having countable tightness.

(2) For a topological space $X$ with unique sequential limits, $X$ is sequential iff $X$ is countably sequential and WACP.

Theorem 2.10. Every countably sequential and countably AP space having countable tightness is Fréchet-Urysohn.

Proof. Let $A$ be a subset of a countably sequential and countably AP space $X$ having countable tightness with $\bar{A} \backslash A \neq \emptyset$ and $x \in \bar{A} \backslash A$. Then since $X$ has countable tightness, there exists a countable subset $C_{x}$ of $A$ such that $x \in \overline{C_{x}}$. Since $X$ is countably AP, there exists a subset $B_{x}$ of $C_{x}$ such that $\overline{B_{x}}=B_{x} \cup\{x\}$. Since $X$ is countably sequential, there exists a sequence $\left(x_{n}\right)$ of points of $B_{x}$ such that $\left(x_{n}\right)$ converges to $x$. Thus, $X$ is Fréchet-Urysohn.

From Theorem 2.10, we have the following corollaries.

Corollary 2.11. Every sequential and countably AP space is FréchetUrysohn.

Proof. Note that every sequential space is a countably sequential space having countable tightness. Hence, it follows from Theorem 2.10.

From Corollary 2.11 above, we obtain directly that every sequential and AP space is Fréchet-Urysohn (see [1, Corollary 2.2(6)] and [13, Proposition 2.1(6)]).

As a corollary, we obtain that in the class of countably sequential spaces having countable tightness and unique sequential limit, the five properties: Fréchet-Urysohn, countably Fréchet-Urysohn, ACP, AP, and countably AP coincide.

Corollary 2.12. Let $X$ be a countably sequential space having countable tightness and unique sequential limits. Then the following statements are equivalent.

(1) $X$ is Fréchet-Urysohn.

(2) $X$ is countably Fréchet-Urysohn.

(3) $X$ is ACP.

(4) $X$ is AP. 
(5) $X$ is countably AP.

Proof. By definitions, obviously $(1) \Rightarrow(2) \Rightarrow(5)$ and $(1) \Rightarrow(3) \Rightarrow(4) \Rightarrow(5)$. From Theorem $2.10,(5) \Rightarrow(1)$. Thus, it holds.

In [8, Corollary 2.10], the author showed that a countably compact space satisfying one of properties mentioned in Introduction related to sequentiality except for countable tightness is sequentially compact. We show that another necessary and sufficient condition for a countably compact space $X$ to be sequentially compact is countably sequential.

Theorem 2.13. A topological space $X$ is countably compact and countably sequential iff $X$ is sequentially compact.

Proof. It is obvious that every sequentially compact space is countably compact and countably sequential.

Conversely, let $\left(x_{n}\right)$ be a sequence of points of a countably compact and countably sequential space $X$. If the range $\left\{x_{n}: n \in \mathbb{N}\right\}$ of $\left(x_{n}\right)$ is finite, then it is obvious. Hence, assume that $\left(x_{n}\right)$ is regular(i.e., every terms are distinct). Then since $X$ is countably compact (and $T_{1}$ ), there exists an accumulation point of $\left(x_{n}\right)$ in $X$ and so $\overline{\left\{x_{n}: n \in \mathbb{N}\right\}} \backslash\left\{x_{n}: n \in\right.$ $\mathbb{N}\} \neq \emptyset$. Since $X$ is countably sequential, there exist $x \in \overline{\left\{x_{n}: n \in \mathbb{N}\right\}} \backslash$ $\left\{x_{n}: n \in \mathbb{N}\right\}$ and a sequence $\left(y_{n}\right)$ of points of $\left\{x_{n}: n \in \mathbb{N}\right\}$ such that $\left(y_{n}\right)$ converges to $x$. It is easy to show that there exists a subsequence $\left(x_{\phi(n)}\right)$ of $\left(x_{n}\right)$ such that $\left(x_{\phi(n)}\right)$ is also a subsequence of $\left(y_{n}\right)$. Thus, $\left(x_{\phi(n)}\right)$ converges to $x$, and therefore $X$ is sequentially compact.

From Theorem 2.13, we obtain directly the following corollaries.

Corollary 2.14. ([1, P.53, Proposition 3], [6, Proposition 1.10] and $[14$, p.590, 5.3]) Every countably compact and sequential space is sequentially compact.

Proof. It is obvious.

Note that in Example 2.2(1), the space $X=\left[0, \omega_{1}\right]$ is sequentially compact (see [14]), but not sequential. Hence, we know that the converse of Corollary 2.14 above need not be true in general.

We recall a topological space $X$ is $S C$-closed [9] iff every sequentially compact subset of $X$ is closed.

Corollary 2.15. Every countably compact, countably sequential and SC-closed space is sequential.

Proof. Note that every sequentially compact and SC-closed space is sequential (see [9, Theorem 2.4]). Thus, it is obvious. 
And we study some necessary and sufficient conditions for a countably sequential space $X$ to have unique sequential limits.

Theorem 2.16. Let $X$ be a countably sequential space. Then the following statements are equivalent.

(1) $X$ has unique sequential limits.

(2) Every countable and compact subset of $X$ is closed.

(3) Every countable and countably compact subset of $X$ is closed.

(4) Every countable and sequentially compact subset of $X$ is closed.

Proof. (3) $\Rightarrow(2)$ and $(3) \Rightarrow(4)$ are obvious.

$(1) \Rightarrow(3)$ : Suppose that there exists a countable and countably compact subset $K$ of a countably sequential space $X$ with unique sequential limits which is not closed. Then clearly $K$ is infinite. Let $K=\left\{x_{n}: n \in\right.$ $\mathbb{N}$ \}. Since $X$ is countably sequential and $K$ is countable and non-closed, there exist $x \in \bar{K} \backslash K$ and a sequence $\left(y_{n}\right)$ of points of $K$ such that $\left(y_{n}\right)$ converges to $x$. Since $X$ has unique sequential limits, $\left\{y_{n}: n \in \mathbb{N}\right\} \cup\{x\}$ is closed and hence $x$ is the only one accumulation point of $\left\{y_{n}: n \in \mathbb{N}\right\}$. Since $K$ is countably compact and $\left(y_{n}\right)$ is a sequence of points of $K$, we have $x \in K$. This is a contradiction.

$(3) \Rightarrow(1)$ : Suppose that there exists a sequence $\left(x_{n}\right)$ of points of a countably sequential space $X$ satisfying property (3) such that $\left(x_{n}\right)$ converges to $x$ and $y$ with $x \neq y$. Then obviously $\left\{x_{n}: n \in \mathbb{N}\right\} \cup\{x\}$ is countable and non-closed. Hence, by (3), $\left\{x_{n}: n \in \mathbb{N}\right\} \cup\{x\}$ is not countably compact. On the other hand, from the fact that $\left(x_{n}\right)$ converges to $x$, it follows that $\left\{x_{n}: n \in \mathbb{N}\right\} \cup\{x\}$ is compact and hence countably compact. This is a contradiction.

$(2) \Rightarrow(1)$ : Suppose that there exists a sequence $\left(x_{n}\right)$ of points of a countably sequential space $X$ satisfying property (2) such that $\left(x_{n}\right)$ converges to distinct points $x$ and $y$. Let $K=\left\{x_{n}: n \in \mathbb{N}\right\} \cup\{x\}$. Then clearly $K$ is countable, compact, and non-closed. Since $K$ is not closed, by (2), $K$ must be non-countable or non-compact. This is a contradiction.

$(4) \Rightarrow(3)$ : It follows from Theorem 2.13 .

Finally, we introduce a new property of a topological space which is another generalization of a sequential space and show that the property is a necessary and sufficient condition for a topological space $X$ having countable tightness to be sequential.

Consider the following property of a topological space $X$.

(*) For each countable subset $C$ of $X, \bar{C}$ is sequential. 
It is not difficult to see that every sequential space is $(*)$ and every topological space with $(*)$ is countably sequential.

Theorem 2.17. A topological space $X$ having countable tightness is $(*)$ iff $X$ is sequential.

Proof. Obviously, every sequential space has countable tightness and $(*)$.

Conversely, let $A$ be a subset of a topological space $X$ having countable tightness and $(*)$ with $\bar{A} \backslash A \neq \emptyset$. Let $x \in \bar{A} \backslash A$. Then since $X$ has countable tightness, there exists a countable subset $C_{x}$ of $A$ such that $x \in \overline{C_{x}}$. Let $B=\overline{C_{x}} \cap A$. Then clearly $C_{x} \subset B \subset \overline{C_{x}}$ and hence $\bar{B}=\overline{C_{x}}$ and $x \in \bar{B} \backslash B$. Since $X$ is $(*), \overline{C_{x}}$ is sequential and so $\bar{B}$ is sequential. Hence, there exist $y \in \bar{B} \backslash B(\subset \bar{A} \backslash A)$ and a sequence $\left(x_{n}\right)$ of points of $B(\subset A)$ such that $\left(x_{n}\right)$ converges to $y$. Thus, $X$ is sequential.

Remark 2.18. (1) It is clear that if every countable subset of a topological space $X$ is closed, then $X$ is $(*)$. Hence, in Example 2.2(3), the space $X$ is $(*)$. But, $X$ is not WAP and does not have countable tightness, and hence it is not sequential. In Example 2.2(2), since the space $X$ is countable, obviously $X$ has countable tightness. But, $X$ is not countably sequential, and hence it is not $(*)$. And suppose that every WAP space is $(*)$. Then since every WACP space is WAP, it follows that every WACP space is sequential from Theorem 2.7. It is impossible. Hence, a WAP space need not be $(*)$ in general. Thus, we know that being WAP, having countable tightness and being $(*)$ are independent (even though Hausdorff).

(2) Still there is a very natural question left open: Is a countably sequential WAP space $(*)$ ?

Acknowledgement. The author wish to thank the referee for his/her kind detailed comments and suggestions in improving the exposition of the paper.

\section{References}

[1] A. V. Arhangel'skii and L. S. Pontryagin (Eds.), General Topology I, Encyclopaedia of Mathematical Sciences, vol. 17, Springer-Verlage, Berlin, 1990.

[2] A. Bella, On spaces with the property of weak approximation by points, Comment. Math. Univ. Carolinae 35(2) (1994), 357-360.

[3] A. Bella and I. V. Yaschenko, On AP and WAP spaces, Comment. Math. Univ. Carolinae 40(3) (1999), 531-536.

[4] M. H. Cho, J. Kim and M. A. Moon, Examples and functions around AP and WAP spaces, Commun. Korean. Math. Soc. 23(3) (2008), 447-452. 
[5] J. Dugundji, Topology, Allyn and Bacon, Inc., Boston, 1970.

[6] S. P. Franklin, Spaces in which sequences suffice, Fund. Math. 57 (1965), 107-115.

[7] S. P. Franklin, Spaces in which sequences suffice II, Fund. Math. 61 (1967), 51-56.

[8] W. C. Hong, Generalized Fréchet-Urysohn spaces, J. Korean Math. Soc. 44(2) (2007), 261-273.

[9] W. C. Hong, On spaces in which compact-like sets are closed, and related spaces, Commun. Korean. Math. Soc. 22(2) (2007), 297-303.

[10] W. C. Hong, On spaces which have countable tightness and related spaces, Honam Math. J. 34(2) (2012), 199-208.

[11] J. Penlant, M. G. Tkachenko, V. V. Tkachuk, and R. G. Wilson, Pseudocompact Whyburn spaces need not be Fréchet, Proc. Amer. Math. Soc. 131(10) (2002), 3257-3265.

[12] L. A. Steen and J. A. Seebach, Jr., Counterexamples in topology, Springer-Verlag, Berlin, 1978.

[13] V. V. Tkachuk and I. V. Yaschenko, Almost closed sets and topologies they determine, Comment. Math. Univ. Carolinae 42(2) (2001), 395-405.

[14] J. E. Vaughan, Countably compact and sequentially compact spaces, Handbook of Set-Theoretic Topology, North-Holland, Amsterdam, New York, Oxford (1984), 569-602.

[15] A. Wilansky, Topology for Analysis, Ginn and Company, 1970.

\section{Woo Chorl Hong}

Department of Mathematics Education, Pusan National University, Pusan 609-735, Korea.

E-mail: wchong@pusan.ac.kr

Seonhee Kwon

Department of Mathematics, Pusan National University, Pusan 609-735, Korea.

E-mail: shkwon307@pusan.ac.kr 